\title{
Grazing-Incidence Texture Tomography of a Patterned Organic Semiconductor Film
}

\author{
Detlef-M. Smilgies ${ }^{1,2 *}$, Margaret K. A. Koker ${ }^{3}$, Ruipeng $\mathrm{Li}^{4}$, Leo Shaw ${ }^{5}$, and Zhenan Bao ${ }^{5}$ \\ ${ }^{1}$ Materials Science and Engineering Program and Center for Advanced Microelectronics Manufacturing \\ (CAMM), Binghamton University, Binghamton NY 13902 \\ ${ }^{2}$ R. F. Smith School of Chemical and Biomolecular Engineering, Cornell University, Ithaca, NY 14853 \\ ${ }^{3}$ Department of Physics, Lawrence University, Appleton WI 54911 \\ ${ }^{4}$ NSLS-II, Brookhaven National Laboratory, Upton, NY 11973 \\ ${ }^{5}$ Department of Chemical Engineering, Stanford University, Stanford, CA 94305-4125 \\ *email: dsmilgie@binghamton.edu
}

\begin{abstract}
The use of grazing-incidence scattering methods for the characterization of 2D patterned organic thin films is limited due to the elongated 1D footprint of the X-ray beam on the sample. However, this characteristic feature can be turned into an advantage, when combined with tomographic reconstruction. In this pilot study we show how the use of a chosen texture reflection and a diffuse reflectivity signal can each provide 2D images of the deposits, simultaneously revealing the organic film's crystal orientation and the location of the metal electrodes.
\end{abstract}

\section{Introduction}

Thin films of organic semiconductors (OSC) have been studied extensively by grazing-incidence wideangle X-ray scattering (GIWAXS), an effective and highly sensitive method that, in addition, avoids excessive radiation damage. Grazing-incidence scattering reduces background signals from the substrate by confining the penetration of the X-ray beam into the substrate when the incidence angle is chosen to be below the substrate critical angle. However, the grazing-incidence geometry poses challenges for spatially resolved measurements, as the beam footprint is extended along the sample in the direction parallel to the beam. Even using an X-ray microbeam $20 \mu \mathrm{m}$ in width limits scanning $\mu$ GIWAXS to probe a scattering area of $20 \mu \mathrm{m}$ by $6000 \mu \mathrm{m}$. Thus only one dimensional patterns can be studied, as was shown in previous publications (Li et al., 2012; Ward et al., 2014; Niazi et al., 2016). However, for X-ray 
studies of two-dimensionally patterned thin films, which are important for actual devices, $\mu$ GIWAXS by itself is of limited value. Here, we devise tomographic methods to resolve 2D patterned samples with high spatial resolution. By using both sample translation (perpendicular to the X-ray beam) and in-plane sample rotation, we can process the resulting $X$-ray diffractograms into 2D maps or sinograms from which the 2D distribution of the diffraction intensity for a chosen reflection or range of $q_{z}$-values can be computationally reconstructed. In other words, by tracking any texture reflection $\left(q_{x y} \approx 0\right)$ with sufficient diffraction intensity, this technique generates a map that identifies and highlights local regions of a specific crystalline material and orientation.

A characteristic feature of small organic molecules is that their crystallites typically assume a limited number of crystallographic planes that are oriented parallel to the substrate, i.e. they have a welldefined texture. This property is particularly important for OSCS - we have shown previously that mobility in an organic field-effect transistor (OFET) is optimized, if only one texture plane is present (Li et al., 2012). Hence, diffraction imaging of the thin film in light of the texture reflection reveals both the texture map as well as the amount of crystalline material present in each pixel of the reconstructed image. Texture reflections by their very nature are always visible at any azimuth angle and are thus suitable for tomographic measurements.

\section{Experimental}

\subsection{Sample preparation}

The sample was patterned using the CONNECT method, which involves "controlled OSC nucleation and extension for circuits" (Park, et al., 2015) and uses differential surface energy and solution shearing to simultaneously generate patterned and precisely registered OSC thin films within the channel region between electrodes. First, gold electrodes were deposited using photolithography, and then the remaining silicon oxide areas were functionalized with octadecyltrichlorosilane (OTS), while the gold electrodes were functionalized with thiolphenol. The substrates were then coated using solution shearing, a type of blade coating, with a solution of the OSC dissolved in $p$-dichlorobenzene. The surface energy on the functionalized gold strips is more favorable for the OSC solution, while the OSC solution dewets from the OTS functionalized regions. After drying a solid OSC film was preferentially deposited on and between the gold structures in the $2 \mu \mathrm{m}$ channel, thus bridging the source and drain electrodes and yielding a patterned array of field-effect transistors.

A film coated with 6,13-bis(triisopropylsilylethynyl)pentacene (TIPS-Pn) was used to demonstrate our method. Because of coating instabilities this film had some macroscopic imperfections, where thicker deposits occurred, as seen in the optical micrograph in Figure 1. TIPS-pn deposits are known to be highly textured with the (001) lattice planes parallel to the substrate (Park et al., 2015). Thus, the (001) texture reflection of the material could be detected at any substrate azimuth angle irrespective of in-plane grain orientation. 


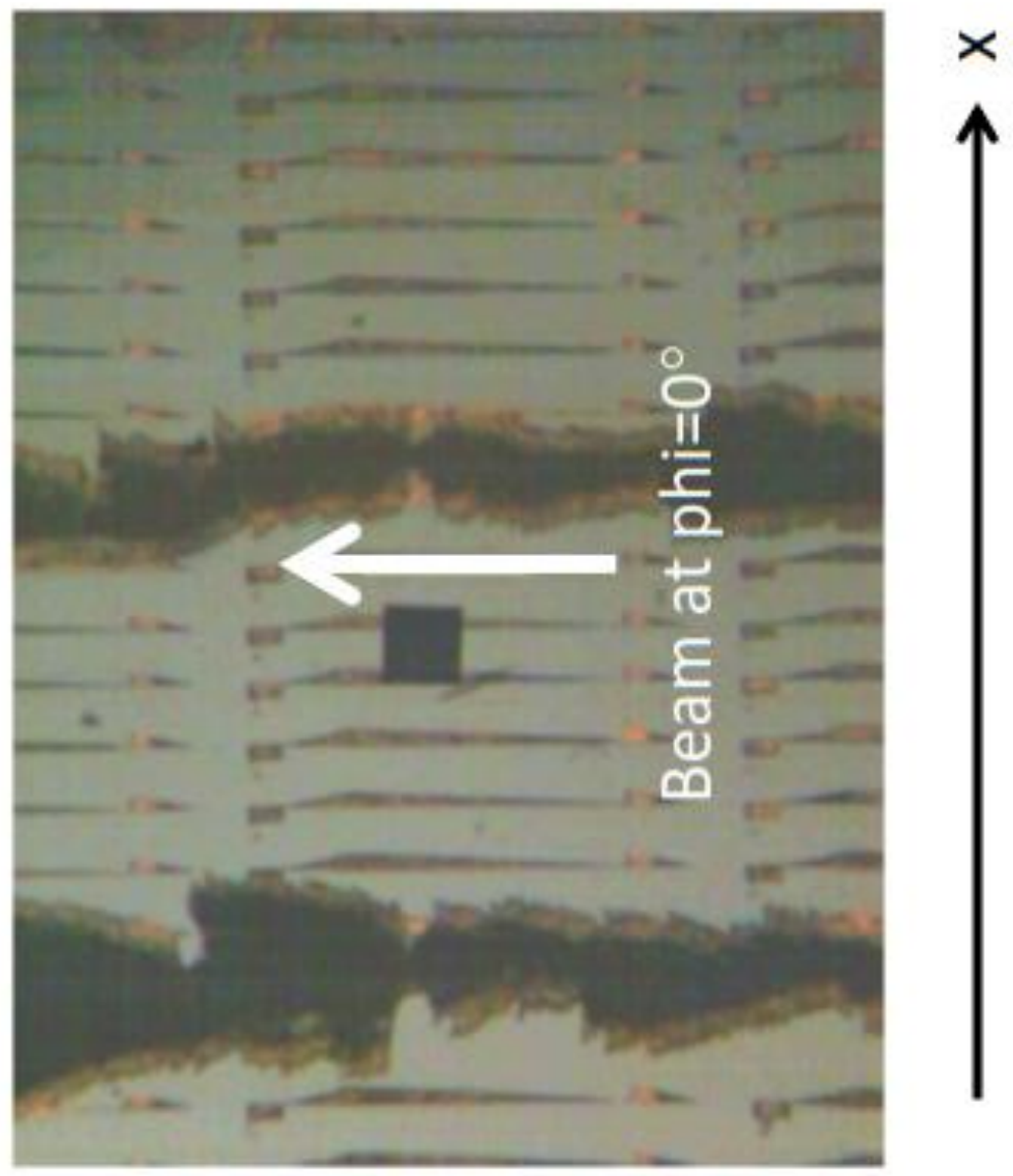

Figure 1. Micrograph of device sample, as taken with the sample microscope at the beamline. Two thicker deposits can be discerned which were due to a coating instability. The spacing between adjacent gold electrodes is $300 \mu \mathrm{m}$. Beam direction (white arrow) and scanning direction $\mathrm{x}$ (black) are indicated.

\subsection{Microbeam characterization}

Proof-of-principle measurements were performed at D1 station using a photon energy of $10.6 \mathrm{keV}$. The $X$-ray beam from a high-flux multilayer monochromator ( $\mathrm{MoB}_{4} \mathrm{C}, 3.0 \mathrm{~nm}$ period, $1.5 \%$ bandwidth) was focused with a single-bounce X-ray focusing capillary to a beam size of $15 \mu \mathrm{m} \times 15 \mu \mathrm{m}$ (Li, Cornaby, Kamperman \& Smilgies, 2011). The microbeam was characterized using a polished cadmium tungstate crystal under a $2^{\circ}$ incidence angle. The cadmium tungstate crystal produced bright blue-white fluorescence. A calibrated optical microscope with variable zoom (Navitar) mounted above the sample was used to characterize the microbeam image parallel and perpendicular to the beam. From the image size along the beam direction, the vertical size of the microbeam could be determined, which then was 
used to estimate the footprint of the X-ray microbeam on the sample under grazing incidence ( $\mathrm{Li}$, Cornaby, Kamperman \& Smilgies, 2011). At an incident angle of $0.15^{\circ}$ which is just above the film critical angle, the length of the footprint is thus about $10 \mathrm{~mm}$.

\subsection{Data collection}

Data were collected using a home-built sample goniometer and a fast pixel array detector (Dectris Pilatus 3 200k, Switzerland) with a read-out time of less than $5 \mathrm{~ms}$. The sample goniometer facilitated rotations to control the incident angle $(\theta)$, the azimuth angle $(\phi)$, and the sideways tilt $(\chi)$, as well as horizontal $(x)$ and vertical $(z)$ translations. A small manual goniometer with crossed arcs was mounted on the azimuth rotation $\phi$ of the main goniometer, and laser alignment was used to line up the sample surface perpendicular to the $\phi$ axis. The sample microscope was used to identify the rotation center. Finally, a polished fluorescent cadmium tungstate crystal was used to find the position of the microbeam, and the rotation center was carefully lined up with the microbeam position, defining the zero point of the $x$-translation.
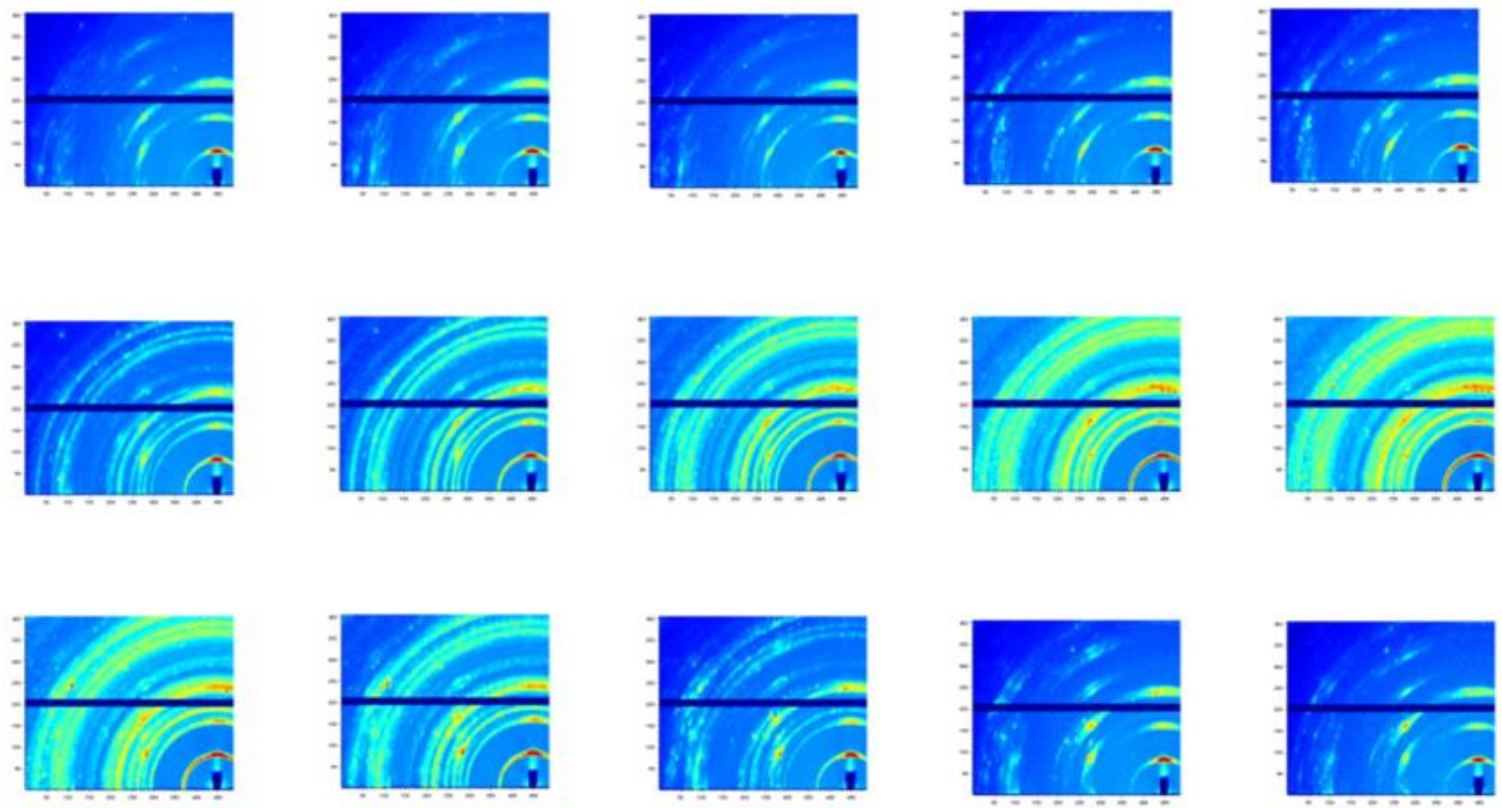

Figure 2. Representative sample images collected on a partial $x$-scan at zero azimuth. The patterns reveal a lot of structural variation.

For measurements, sample height $z$ and incident angle $\theta$ were carefully aligned. The incident angle was calibrated by measuring the reflectivity from the sample across the critical angle of the silicon substrate, which at this photon energy equaled $0.17^{\circ}$. We chose an incident angle of $0.15^{\circ}$ which is just above the critical angle of the film material, but below the critical angle of the gate oxide $\left(0.17^{\circ}\right)$ and the gold 
contacts $\left(0.4^{\circ}\right)$. The parasitic tilt of the sample stage was corrected with the $\chi$ arc such that the sample was perfectly illuminated over the scanning range in $\mathrm{x}$. Finally some test images were taken at a variety of azimuths to ensure that the sample was properly aligned in the beam while rotating the sample around the surface normal.

We scanned the sample in steps of $20 \mu \mathrm{m}$ corresponding roughly to the beam width over a range of \pm 5 $\mathrm{mm}$ with regard to the rotation center, i.e. the scans had no overlap between illuminated areas. After the $x$-scan, the sample azimuth $\phi$ was increased in steps of $1^{\circ}$, and the sample was scanned again in the $x$-direction. This procedure was repeated until scans covering $180^{\circ}$ in azimuth were obtained. All in all, about 45,000 diffraction images were collected with an exposure time of $0.2 \mathrm{~s}$ per image (Figure 2).

\subsection{Data analysis}

A region of interest (ROI) around the out-of-plane texture reflection was defined (see Figure 3, left panel), and the intensity was integrated using our custom software, to yield the sinogram shown in Figure 3, right panel. The background scattering was determined by fitting a plane to the edges of the $\mathrm{ROI}$ and subtracted. The integration of the full series of detector images took about 3 hours on a standard laptop and using a home-built Scilab script.
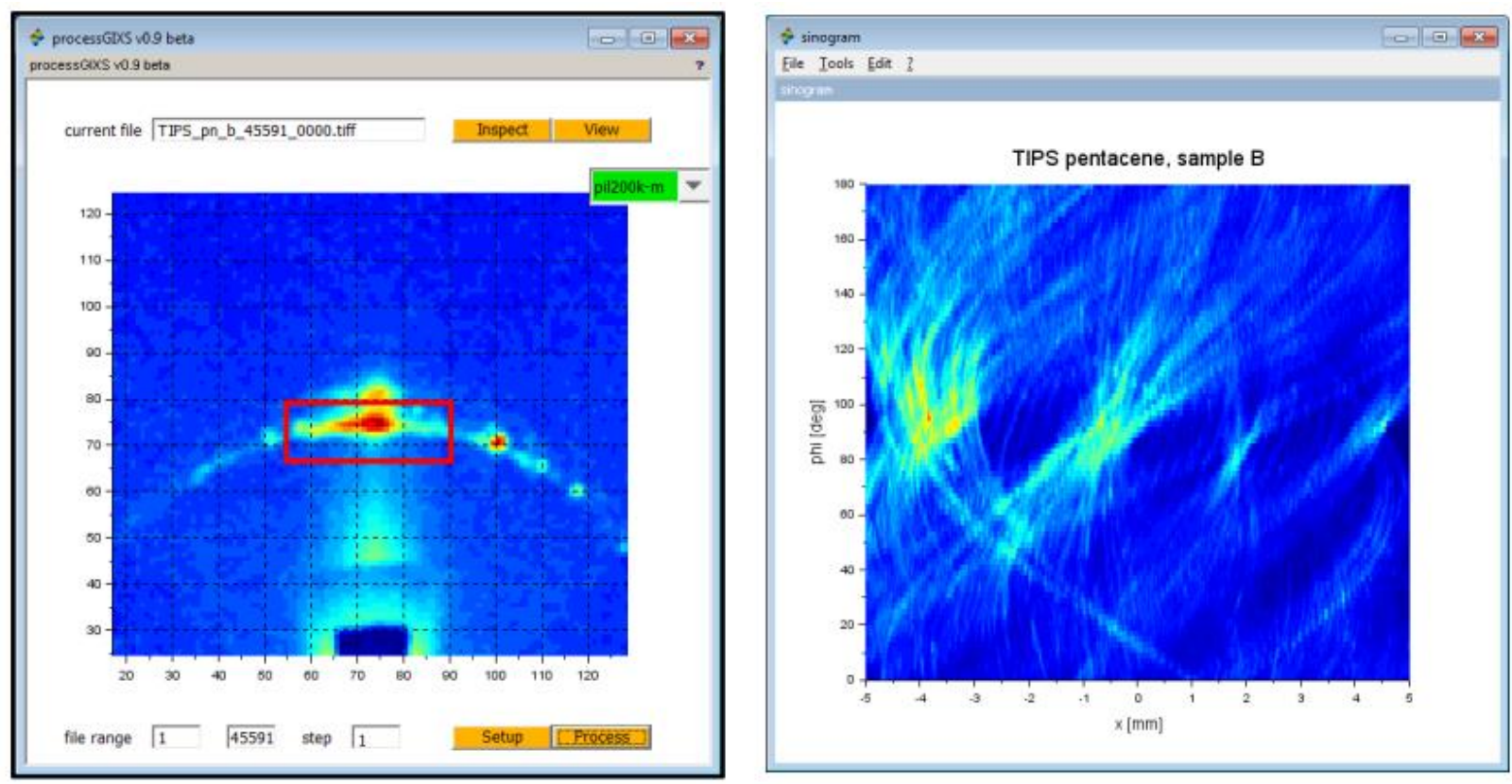

Figure 3. Chosen range-of-interest for the (001) texture reflection (red box in left panel) and associated sinogram (right panel).A 
For the tomographic reconstruction, we used a custom program based on Scilab scripts published by Kai-Meng Hock (2010), which are based on the book by Kak and Slaney (1988). Additional filters (SheppLogan, Hamming, Butterworth) were added, as well as intensity slicing. Calculations were performed on a desktop personal computer, and image reconstruction took a couple of seconds. The reconstruction in Figure 4 shows the resolved detail with bright areas corresponding to areas covered with TIPS-pn with a (001) texture. A Shepp-Logan filter was used and the intensity slice was chosen from $30 \%$ to $70 \%$ of the maximum intensity, in order to enhance the detail.

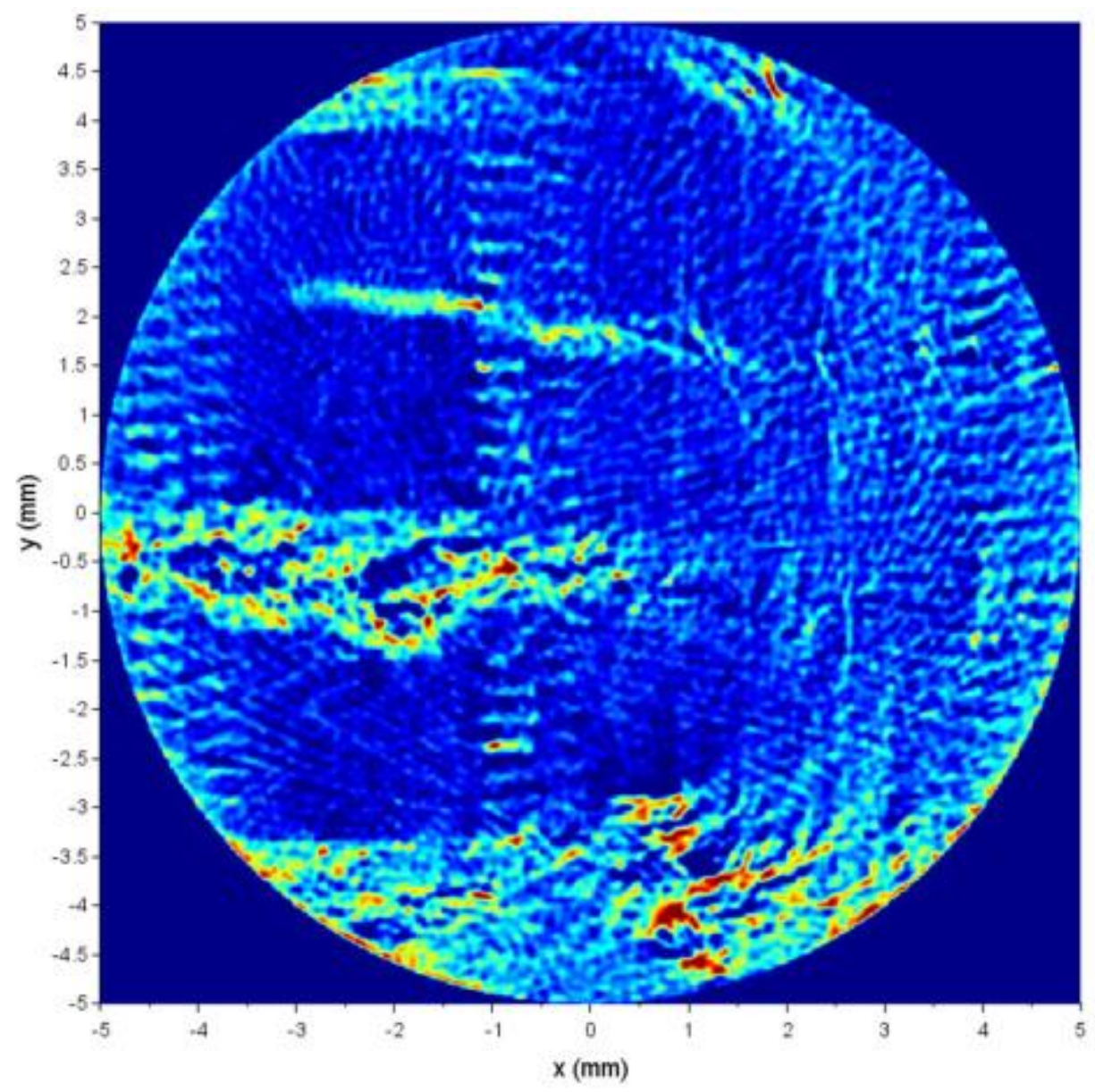

Figure 4. Filtered back-reflection tomographic reconstruction of the organic film in the light of the TIPSpn (001) reflection. A disk of $10 \mathrm{~mm}$ in diameter in the center part of the sample is obtained. Regions of high intensity (red) correspond to crystallites with a (001) orientation and thus indicate the presence of OSC. The location of thick deposits due to a coating instability are also clearly shown (middle and bottom). A fainter signal is obtained from the gold contacts. The finer gold features with the transistor structures were not resolved, however, OSC material was found on the contact pads. 
As gold texture reflections were outside the range of the detector, we set a range of interest around the diffuse reflectivity signal just above the beamstop in order to localize the gold contacts, which reflect the beam more strongly than the uncoated areas due to their higher electron density and related higher critical angle. The latter method is related to reflectivity tomography as recently demonstrated by InnisSamson, Mizusawa, \& Sakurai (2011) as well as GISAXS tomography (Kuhlmann et al., 2009; Ogawa et al., 2015). This diffuse signal resulted in a sinogram with fine features and good contrast (Figure 5).
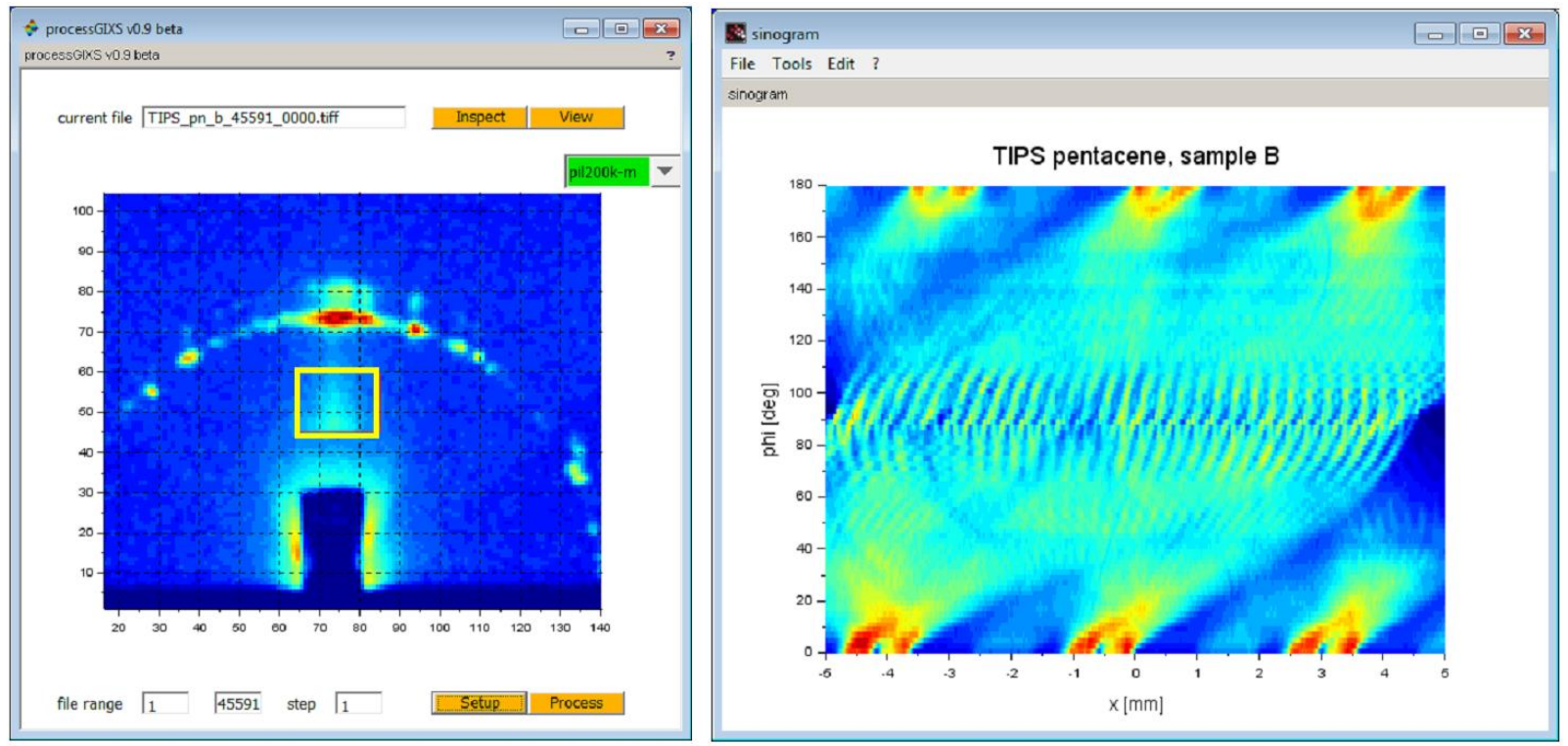

Figure 5. Range-of-interest (yellow box) around the diffuse scattering above the beamstop and associated sinogram.

The reconstruction of the gold scattering yielded a high-quality image which reproduced the fine gold contacts (Figure 6). This means that we have essentially achieved the proper resolution. However the texture data were of less quality than data collected using diffuse reflectivity tomography. A possible reason for this is the large scattering power of gold compared to the OSC. Reducing the beam size to 5 $\mu \mathrm{m}$ should have brought out more detail as well. Moreover, there may have been some shadowing of the beam by the thick deposits; the inhomogeneous sinogram of the (001) texture reflection is an indication for this potential problem. Nonetheless, the image reconstruction yielded information about a major part of the illuminated sample area. 


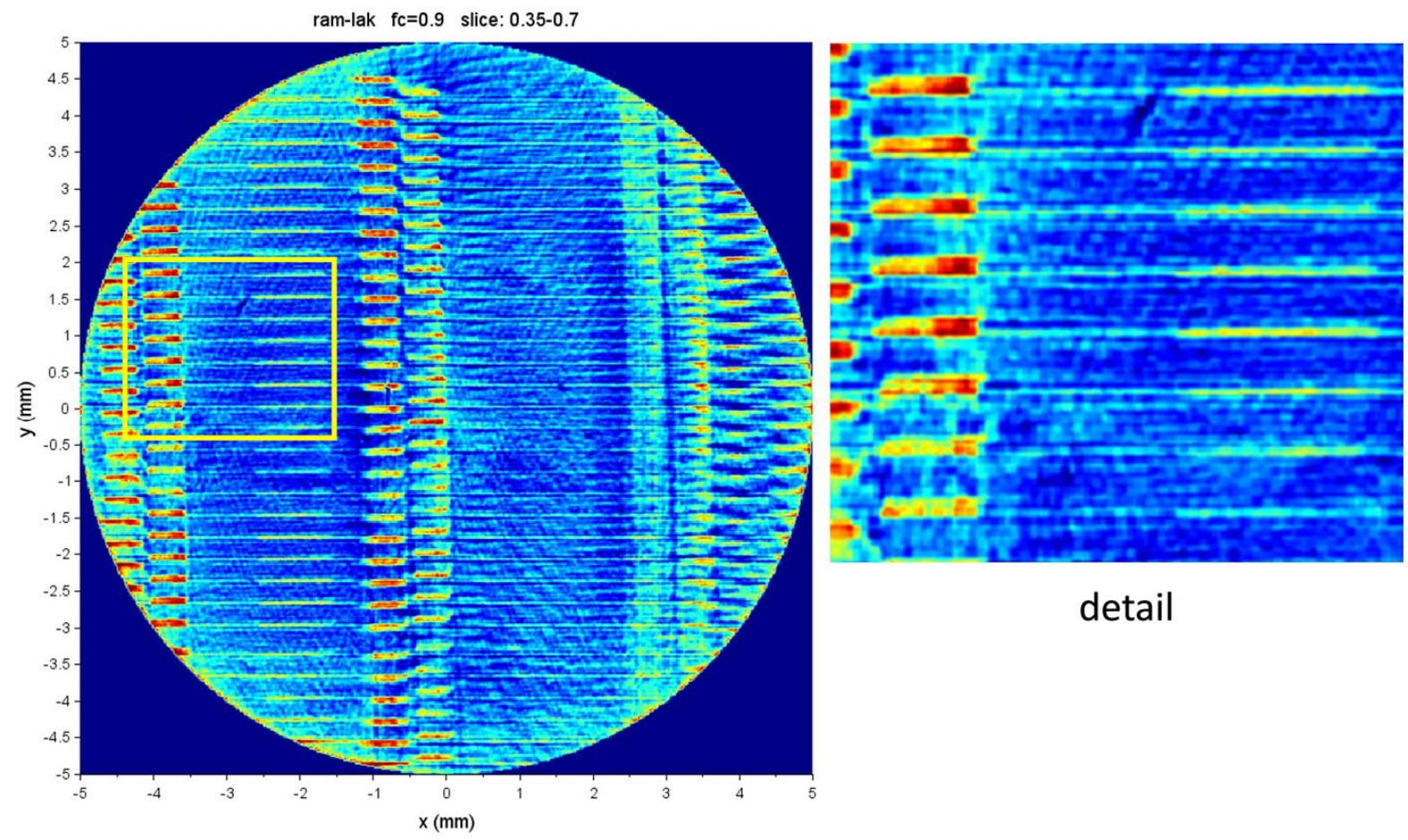

Figure 6. Reconstruction of the diffuse scattering. The detail shows that most of the fine gold structures (the red regions of high intensity) within the 20 micron resolution are reproduced.

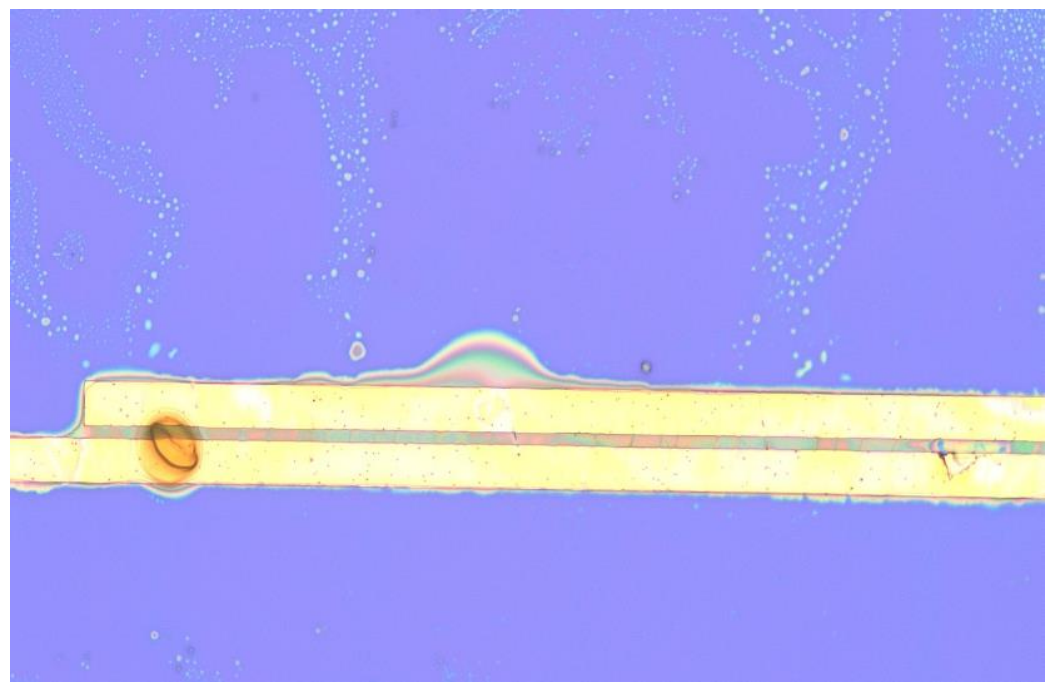

Figure 7. High-resolution optical micrograph of a single field-effect transistor. The gold electrode strips have a width of $10 \mu \mathrm{m}$. The silicon substrate coated with a $300 \mathrm{~nm}$ gate oxide appears blue. The transparent OSC materials is visible via its interference colors and covers both gold strips and the gap well. The upper part shows some microdroplets, most likely due to incomplete dewetting of the OTSmasked silicon oxide. 
We compared the tomography results with images taken with a high-resolution Keyence metrology microscope. These measurements revealed that the gold strips had a width of only $10 \mu \mathrm{m}$ with a gate channel of $2 \mu \mathrm{m}$ width. Nonetheless, we can clearly identify the gold electrodes in the tomogram, while the gate channel was below our resolution, as given by the full-width at half-maximum of the $15-\mu \mathrm{m}$ microbeam. Figure 7 shows that the OSC bridged the gate channel, yielding a functional field-effect transistor. Due to optical interference effects the transparent OSC material shows up as colorful deposits on a blue background which is given by the $300 \mathrm{~nm}$ gate oxide of the substrate, and is itself an interference color. The interference fringes due to the OSC film indicate a significant thickness variation. On top of the gold electrodes the OSC film is hard to discern.

\section{Conclusion}

We have introduced two tomography variants, grazing-incidence texture tomography (GITT) and diffuse reflectivity tomography (DRT). GITT uses a texture reflection for tomographic reconstruction and is a useful tool to characterize the morphology of patterned thin films. The tomographic reconstruction yields the 2D distribution of the thin film target material with a specific orientation, irrespective of inplane grain orientation. GITT differs from diffraction tomography where it is assumed that each voxel produces a complete powder pattern (Bleuet, Welcomme, Dooryhee, Susini, Hodeau, \& Walter, 1988). GITT is a powerful method when multiple texture planes exist, and this way different orientational domains of the thin film can be selectively imaged. Microbeam scanning can be extended to reach micrometer or even sub-micrometer resolution, suitable for future characterization of flexible organic circuits that find application as biomedical sensors (Schwartz et al., 2013, Rogers et al., 2018), fitness sensors (Amjadi et al, 2016), and general flexible sensors for automobile and aerospace applications. Texture tomography can conveniently be combined with reflectivity tomography using the same data set, in order to locate structures containing chemical elements with high electron density. Combining the information from GITT and DRT based on the same series of diffractograms provides a way to detect how molecular deposits are distributed with respect to metal contacts.

\section{Acknowledgement}

This work is based upon research conducted at the Cornell High Energy Synchrotron Source (CHESS) which is supported by the National Science Foundation under award DMR-1332208. High-resolution optical microscopy images were obtained at the Center for Advanced Microelectronics Manufacturing (CAMM) at Binghamton University. 


\section{References}

Amjadi, M., Kyung, K.-U., Park, I., Sitti, M. (2016). Adv. Funct. Mater. 2016, 26, 1678-1698.

Bleuet, P., Welcomme, E., Dooryhee, E., Susini, J., Hodeau, J.-L., \& Walter, P. (1988). Probing the structure of heterogeneous diluted materials by diffraction tomography. Nat. Mater. 7, 468-472.

Hock, K. M., http://hep.ph.liv.ac.uk/ hock/tomography/Scilab/index.html (last update 6/2010).

Innis-Samson, V. A., Mizusawa, M., \& Sakurai, K. (2011). X-ray Reflection Tomography: A New Tool for Surface Imaging. Anal. Chem. 83, 7600-7602.

Kak, A., \& Slaney, M. (1988). Principles of Computerized Tomographic Imaging. New York: IEEE Press.

Kuhlmann, M., Feldkamp, J. M., Patommel, J., Roth, S. V., Timmann, A., Gehrke, R., et al. (2009). Grazing Incidence Small-Angle X-ray Scattering Microtomography Demonstrated on a Self-Ordered Dried Drop of Nanoparticles. Langmuir 25, 7241-7243.

Li, R., Cornaby, S., Kamperman, M., \& Smilgies, D.-M. (2011). Nanocomposite Characterization on Multiple Length Scales Using $\mu$ SAXS. J. Synchrotron Rad. 18, 697-701.

Li, R., Ward, J. W., Smilgies, D.-M., Payne, M. M., Anthony, J. E., Jurchescu, O. D. (2012). Direct structural mapping of organic field-effect transistors reveals bottlenecks to carrier transport. Adv. Mater., 24, 5553-5558.

Niazi, M. R., Li, R., Abdelsamie, M., Zhao, K., Anjum, D. H., Payne, M. M., et al. (2016). Contact-Induced Nucleation in High-Performance Bottom-Contact Organic Thin Film Transistors Manufactured by Large-Area Compatible Solution Processing. Adv. Funct. Mater. 26, 2371-2378.

Ogawa, H., Nishikawa, Y., Fujiwara, A., Takenada, M., Wang, Y.-C., Kanayaa, T., et al. (2015). Visualizing patterned thin films by grazing-incidence small-angle $\mathrm{X}$-ray scattering coupled with computed tomography. J. Appl. Cryst. 48, 1645-1650.

Park, S., Giri, G., Shaw, L., Pitner, G., Ha, J., Koo, J., et al. (2015). Large-area formation of self-aligned crystalline domains of organic semiconductors on transistor channels using CONNECT. Proc. Natl. Acad. Sci. 112, 5561-5566.

Rogers, J., Malliaras, G. \& Someya, T. (2018). Biomedical devices go wild. Sci. Adv. 4, eaav1889.

Schwartz, G., Tee, B. C.-K., Mei, J., Appleton, A. L., Kim, D. H., Wang, H. \& Bao, Z. (2013). Flexible Polymer Transistors with High Pressure Sensitivity for Application in Electronic Skin and Health Monitoring. Nature Commun. 4, 1-8. 
Ward, J. W., Li, R., Obaid, A., Payne, M. M., Smilgies, D.-M., Anthony, J. E. \& Jurchescu, O. (2014). Rational Design of Organic Semiconductors for Texture Control and Self-Patterning on Halogenated Surfaces. Adv. Funct. Mater. 24, 5052-5058. 